DOE/ER/60721-6

\title{
QUASI-ELASTIC NEUTRON SCATTERING STUDIES OF PROTEIN DYNAMICS
}

\author{
Progress Report
}

November 1, 1992 - May 25, 1993

H. E. Rorschach

Department of Physics

W. M. Rice University

Houston, Texas 77251

May 25, 1993

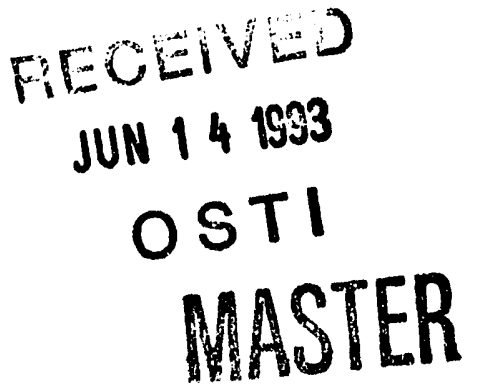

DISTRIBUTION OF THIS DOCUMENT IS UNLIMITED

\section{PREPARED FOR THE U. S. DEPARTMENT OF ENERGY UNDER GRANT NUMBER DE-FG05-88ER60721}

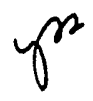

\section{DISCLAIMER}

\begin{abstract}
This report was prepared as an account of work sponsored by an agency of the United States Government. Neither the United States Government nor any agency thereof, nor any of their employees, makes any warranty, express or implied, or assumes any legal liability or responsibility for the accuracy, completeness, or usefulness of any information, apparatus, product, or process disclosed, or represents that its use would not infringe privately owned rights. Reference herein to any specific commercial product, process, or service by trade name, trademark, manufacturer, or otherwise does not necessarily constitute oi imply its endorsement, recommendation, or favoring by the United States Government or any agency thereof. The views and opinions of authors expressed herein do not necessarily state or reflect those of the United States Government or any agency thereof.
\end{abstract}


Since our last technical report, we have obtained important results that shed new light on the study of protein dynamics by quasi-elastic neutron scattering. The experimental results presented in this report were obtained in August, 1992, during a three-week visit at Brookhaven National Laboratory. While there, we obtained two weeks of reactor time on the triple axis instrument $\mathrm{H}-9$ supplied by the cold source. We were able to perform a detailed study of the quasi-elastic spectrum and the Debye-Waller factor for trypsin in powder form, in solution, and in crystals. We also made a preliminary study of myoglobin crystals. In this report, we will sketch a new way to view the results of quasielastic scattering experiments. We will present the data on trypsin and analyze it according to this new picture.

1. Collaborators. These experiments were conducted on the $\mathrm{H}-9$ triple axis spectrometer at Brookhaven National Laboratory (BNL) in collaboration with $\mathrm{H}$. Cao and C. F. Hazlewood of Rice University and S. Shapiro and T. Thurston of BNL.

2. Theoretical background. Due to the large incoherent scattering crcss-section for protons in comparison with all other nuclei, in the scattering of neutrons from proteins, the scattering is predominantly due to the motion of the hydrogen protons. Since the scattering is incoherent, the scattered intensity $S_{S}(Q, \omega)$ is due to the motion of single atoms and is determined by the self-correlation function $G_{s}(r, t)$. This function gives the probablity that an atom undergoes a displacement $r$ in the time $t$. The scattered intensity is then given by (L. van Hove, Phys. Rev. 95, 249, 1954)):

$$
S_{S}(Q, \omega)=\int_{-\infty} \int_{-\infty}^{\infty} \exp (i \omega t) \exp (i Q \cdot r) G_{s}(r, t) d r d t
$$

In quasi-elastic scattering, $S_{S}(Q, \omega)$ is measured near $\omega=0$, so that it is the low frequency components of $G_{S}(r, t)$ that are studied. These motions are on the time scale of $10^{-9}$ to $10^{-11}$ seconds, and are usually due to diffusive types of motion. The high- frequency motions outside of the quasi-elastic range may consist of oscillations, linear or non-linear, and rapid jumps between quasi-equilibrium sites. These motions will affect the quasielastic line by a Q-dependent reduction in the total integrated intensity (J. A. Krumhansl, Proc. in Life Sciences: Protein Structure, Molecular, and Electronic Activity, SpringerVerlag, New York, 1987).

In our quasi-elastic scattering experiments, the integrated intensity is determined from the area of the quasi-elastic peak, after subtracting the inelastic and $\mathrm{D}_{2} \mathrm{O}$ background:

$$
S_{m}(Q)=\int_{-\omega_{q}}^{\omega_{q}} S_{s}(Q, \omega) d \omega
$$

$S_{m}(Q)$ is the measured "Debye-Waller factor"。 The integral is over the range $-\omega_{\mathrm{q}}<\omega<\omega_{\mathrm{q}}$, where $\omega_{q}$ is chosen so as to include the quasi-elastic line but not the high-frequency part of the spectrum. Substituting Eq. (1) in Eq. (2), and integrating over $\omega$, we obtain:

$$
S_{m}(Q)=\int_{-\infty}^{\infty} \int_{-\infty}^{\infty} 2 \frac{\sin \omega_{q} t}{t} \exp (i Q \cdot r) G_{s}(r, t) d r d t
$$


We now suppose that the self-correlation function $G_{s}(r, t)$ is due to two uncorrelated types of motion, one slow on the scale of $\omega_{q}$ and the other fast on this scale:

$$
G_{S}(r, t)=G_{\text {slow }}(r, t) \otimes G_{\text {fast }}(r, t)
$$

where $\otimes$ represents a convolution of the two correlation functions. Substituting Eq. (4) in Eq. (3), the resulting function contains the factor $\frac{\sin \omega_{\mathrm{q}} t}{t} G_{\text {slow }}(r, t)$. This function is nearly constant for $\omega_{\mathrm{q}} t<1$, and it is slowly-varying compared with $\mathrm{G}_{\text {fast }}$, whose component frequencies are large compared to $\omega_{\mathrm{q}}$. In the integral in Eq. (3), $\mathrm{G}_{\text {fast }}$ may therefore be replaced by its time-average:

$$
<G_{\text {fast }}(\mathbf{r}, t)>_{t}=\int \rho\left(\mathbf{r}-\mathbf{r}^{\prime}\right) \rho\left(\mathbf{r}^{\prime}\right) d \mathbf{r}^{\prime}=\rho(\mathbf{r}) \otimes \rho(\mathbf{r})
$$

where $\rho(\mathbf{r})$ is the time-averaged single-particle density function, and $\int \rho(\mathbf{r}) \mathrm{dr}=1$ (S Lovesey, Theory of Neutron Scattering from Condensed Matter, Vol 1, p. 62, Oxford, 1984).

The Fourier transform over $\mathbf{r}$ in Eq. (3) can then be performed, making use of the convolution theorem:

$S_{m}(Q)=\left[\int_{-\infty}^{\infty}\left\langle G_{\text {fast }}(r, t)\right\rangle_{t} \exp (\mathrm{iQ} \cdot r) d r\right] \int_{-\infty}^{\infty} \int_{-\infty}^{\infty} 2 \frac{\sin \omega_{\mathrm{q}} t}{t} G_{\text {slow }}(r, t) \exp (\mathrm{iQ} \cdot \mathbf{r}) \mathrm{dr} \mathrm{dt}(6)$

Substituting Eq. (5) in Eq. (6) and making use of the convolution theorem in the first integral,

$$
\int_{-\infty}^{\infty}<G_{\text {fast }}(\mathbf{r}, \mathrm{t})>_{\mathrm{t}} \exp (\mathrm{iQ} \cdot \mathbf{r}) \mathrm{dr}=1 \int_{-\infty}^{\infty} \rho(\mathbf{r}) \exp (\mathrm{iQ} \cdot \mathbf{r}) \mathrm{d} \mathbf{r} \mathrm{R}^{2}
$$

The term in brackets in Eq. (6) is the square of the "Debye-Waller factor" $\left(D W F=\exp \left(-W_{D}\right)\right)$, and the second term gives the quasi-elastic line. This term is independent of $Q$ and $\omega_{q}$ if $\omega_{\mathrm{q}}$ is large compared to the line-width of the quasi-elastic spectrum associated with the diffusive motion.

In the above form, the DWF is seen to be due to diffraction from the time-averaged density distribution of the high-frequency motion of the scattering particle. If the particle oscillates about an equilibrium position with a $p(r)$ that is Gaussian, then the Fourier transform will be Gaussian, and we obtain the usually form for the DWF:

$$
\mathrm{DWF}=\exp \left(-\mathrm{Q}^{2}<\mathrm{u}^{2}>16\right)
$$

If, however, the motion is non-linear, or it involves rapid jumps between two or more quasi-equilibrium positions, then the DWF will contain Q-dependent diffraction-like terms. In any case, the DWF is the Fourier transform of the density distribution of the time-averaged high-frequency motion. 
Returning to Eq. (1), we see that the quasi-elastic contribution can now be written as:

$$
S_{S}(Q, \omega)=(D W F)^{2} \times \int_{-\infty}^{\infty} \int_{-\infty}^{\infty} \exp (i \omega t) \exp (i Q \cdot r) G_{\text {slow }}(r, t) d r d t
$$

This term will produce a quasi-elastic line that depends on the character of $G_{\text {slow }}(r, t)$. For Brownian diffusion,

$$
G_{\text {slow }}(r, t)=(4 \pi D t)^{-3 / 2} \exp \left(-r^{2} / 4 D t\right)
$$

and the quasi elastic spectrum becomes:

with $\Gamma(Q)=D^{2}$.

$$
\mathrm{S}_{\mathrm{qe}}(\mathbf{Q}, \omega)=\frac{1}{\pi} \frac{\Gamma}{\omega^{2}+(\Gamma)^{2}},
$$

Other types of diffusive motion (e.g. jump diffusion) give similar Lorentzian scattering laws with different forms for $\Gamma(\mathrm{Q})$, which depend on the assumed distribution of jump lengths (P. L. Hall and D. K. Ross, Mol. Phys. 42, 673, 1981; W. Gissler and H. Rother, Physica 50, 380, 1970).

\section{Experimental method.}

Samples:

Dialyzed trypsin. Quasi-elastic scattering studies were conducted on the dry powder, $20 \%$ trypsin- $\mathrm{D}_{2} \mathrm{O}$ solutions, trypsin- $\mathrm{D}_{2} \mathrm{O}$ crystals, and pure $\mathrm{D}_{2} \mathrm{O}$ as a function of temperature.

Dialyzed myoglobin. A preliminary study was conducted on myoglobin- $\mathrm{D}_{2} \mathrm{O}$ crystals at 300K.

\section{Spectrometer scans:}

Quasi-elastic spectra were obtained for $0.5<\mathrm{Q}<2.6 \mathrm{~A}^{-1}$ in the energy transfer range $-0.5<\hbar \omega<0.5 \mathrm{meV}$. The spectrometer resolution function was measured to be Gaussian with a FWHM $=.087 \mathrm{meV}$.

Data Analysis:

Data were analyzed with a fitting function which is the sum of a linear background, a Voight function (convolution of a Lorentzian and a Gaussian) for the trypsin, and a Voight function for the $\mathrm{D}_{2} \mathrm{O}$ contribution for the solution and crystal samples. Leastsquares fits to the fitting parameters were made, from which the line width and line intensity were obtained.

\section{Experimental Data.}

Our most complete set of data was taken on the $20 \%$ trypsin- $\mathrm{D}_{2} \mathrm{O}$ solution at $300 \mathrm{~K}$. Quasi-elastic spectra were taken at $18 \mathrm{Q}$-values for $0.5<\mathrm{Q}<2.6 \mathrm{~A}^{-1}$. Data on the line width $\Gamma(Q)$ are shown in Figure 1. The data for the line width of the trypsin crystal are also shown in this figure. In Figure 2, the integrated intensity is given as a function of $Q$. 
We have data at other temperatures, but it is not given in this report. This other data, however, is consistent with the conclusions that we will present below.

\section{Discussion of Data.}

The dependence of the line width $\Gamma(Q)$ given in Figure 1 is characteristic of a jump diffusion process. The approach to an asymptotic value with increasing $Q$ occurs when the Fourier wavelength becomes comparable to the average jump distance. The asymptotic value of the line width is determined by the reciprocal average jump time. (In Brownian diffusion, there would be no asymptotic leveling off.) We do not have a general theory that explains the maximum in $\Gamma(Q)$, but we believe the maximum and decrease at higher $Q$ is associated with jumps to specific sites with a narrow distribution of jump lengths, as occurs for example in the diffusion of hydrogen in metals (see T. Springer, Quasi-elastic Neutron Scattering for the Investigation of Diffusive Motions in Solids and Liquids, Springer-Verlag, 1972, and Gissler and Rother, op. cit.. ). Such jump motions in the quasi-elastic range are consistent with protein dynamics computer simulations (A. E. Garcia, PRL 68, 2696, 1992.)

The Debye-Wailer plot of intensity vs. $Q$ given in Figure 2 is extremely interesting. This plot does not show the usual typical exponential behaviour in $Q^{2}$ (Eq. (7)), but instead shows diffraction-like structure at a $Q$ of approximately $2 \mathrm{~A}^{-1}$. We believe that this structure can be explained with the theory developed in part 2 . Since the DWF is equal to the Fourier transform of the high-frequency average particle density, this data suggests that there are very rapid discrete jumps that occur in the proton side-chain motion, as well as high-frequency oscillations about an equilibrium position. This type of analysis may prove to be a powerful tool for the study of the local "lattice geometry" of the the rapid jumps that a single particle makes during transitions between microconformational states.

It is possible to relate the DWF plot to the effective single-particle potential (see Krumhansl, op. cit.). In Section 1 above, we showed that the DWF is determined by the Fourier transform of the time-averaged high-frequency density function $\rho(r)$. In the classical limit, this density function can be set equal to the ensemble average of $\rho(\mathbf{r})$, which is given by:

$$
<\rho(\mathbf{r})\rangle_{\text {ensemble }} \propto \exp (-B V(\mathbf{r}))
$$

where $\langle\rho(\mathbf{r})>$ ensemble is the Fourier transforr 1 of the DWF. $V(\mathbf{r})$ is thus given by

$$
V(\mathbf{r}) \propto-\frac{1}{B} \ln (<\rho(\mathbf{r})>\text { ensemble })
$$

We have not yet completed the analysis to reduce our data to give a form for $V(r)$, but preliminary fits to our measured DWF suggest that its form is similar to that proposed by Frauenfelder, et. al. (Frauenfelder, Siglar, and Young, Science 254, 1598, 1991).

\section{Conclusions.}

Our experiments on the quasi-elastic scattering from trypsin solutions and crystals and myoglobin crystals show that information can be obtained concerning the dynamics of the low-frequency diffusive motion from $\Gamma(Q)$, and the geometry of the time-averaged highfrequency motion from the DWF. Analysis of our data shows that the diffusive motion is a jump diffusion between definite lattice sites. The high frequency motion also consists of jump motions which produce diffraction effects in the plot of intensity vs. $Q$. 
Measurements on crystals of trypsin and myoglobin show that the quasi-elastic line is narrowed, suggesting a substantial reduction in the slow diffusive jump motion in the crystalline state compared to the solution.

\section{Suggestions for further study.}

This new interpretation of the quasi-elastic studies opens up a wide range of possibilities.

a. Careful studies of the DWF in the solution and crystal as a function of temperature would be most interesting. As the temperature is lowered, the microconformational states of higher energy will be less populated at lower temperatures, changing the time-averaged density distribution. We have some preliminary data at lower temperatures that shows a more pronounced "diffraction" peak in the DWF plot.

b. We want to complete similar studies on myoglobin. We have made preliminary studies of the line width in the crystalline state, but we have not studied the solution, and we have not made a detailed study of the DWF to study the geometry of the highfrequency motion.

c. The motion studied in the quasi-elastic experiments consists of three components: high-frequency oscillations, high-frequency jumps, and low frequency diffusive motion. The low frequency motion determines the quasi-elastic line shape, and we want to extend our detailed studies of $\Gamma(Q)$ in trypsin to lower temperatures and to complete similar studies in myoglobin to clarify the character of the low-frequency jump diffusion processes. 
Fig.1. Width vs. $Q$ for trypsin solution and crystal at $T=300 \mathrm{~K}$

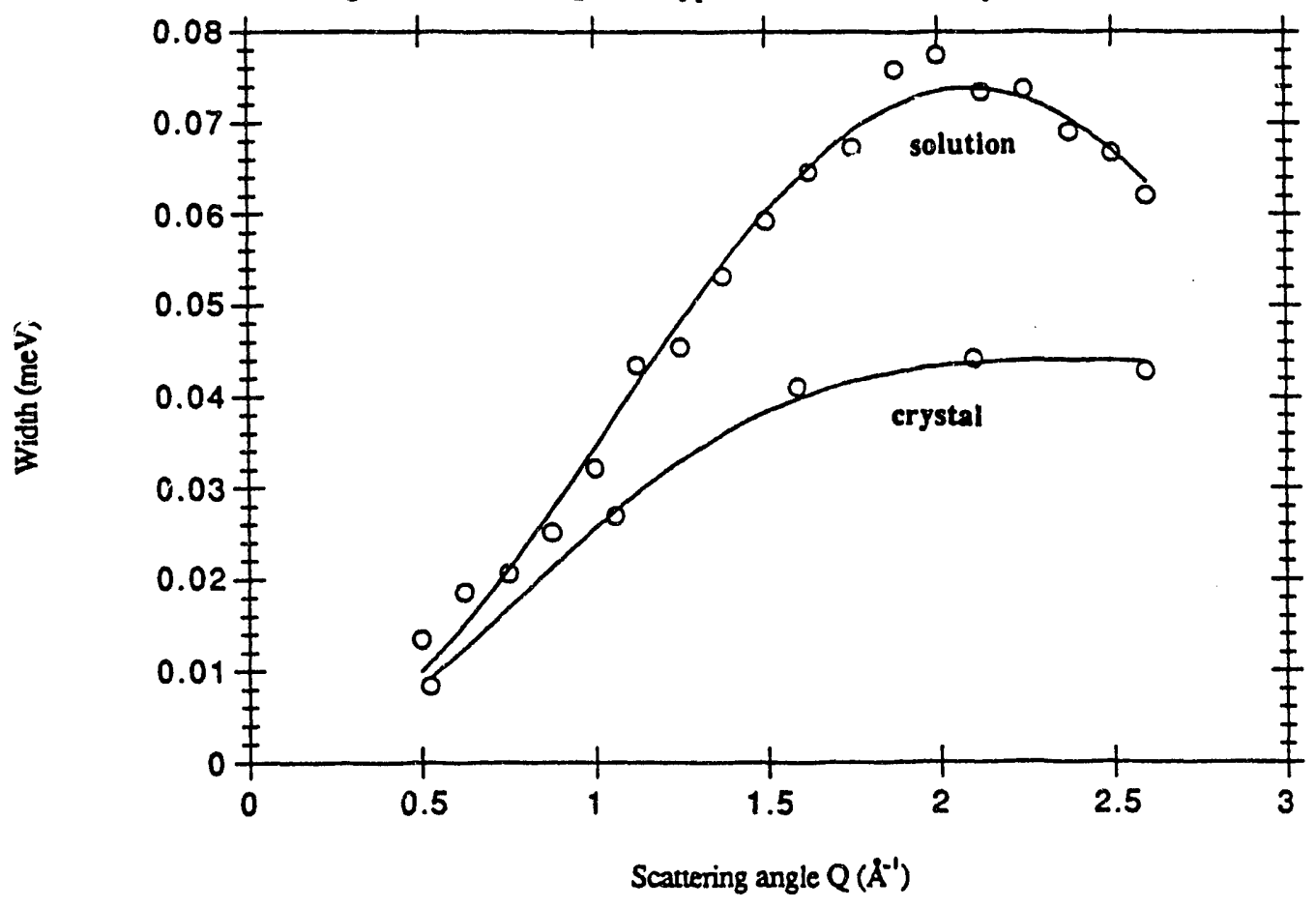

Fig.2. DWF for trypsin solution at $\mathrm{T}=300 \mathrm{~K}$

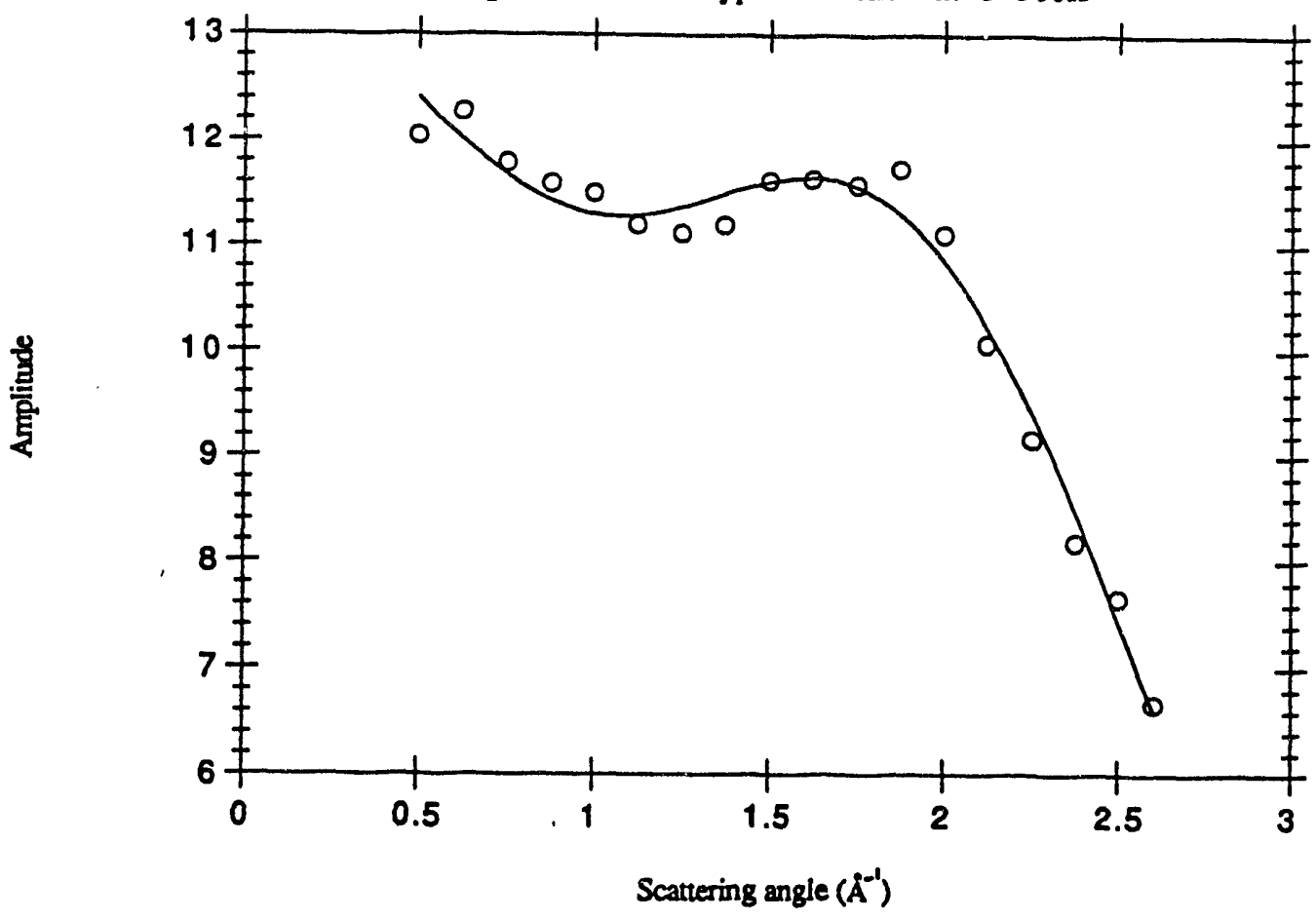



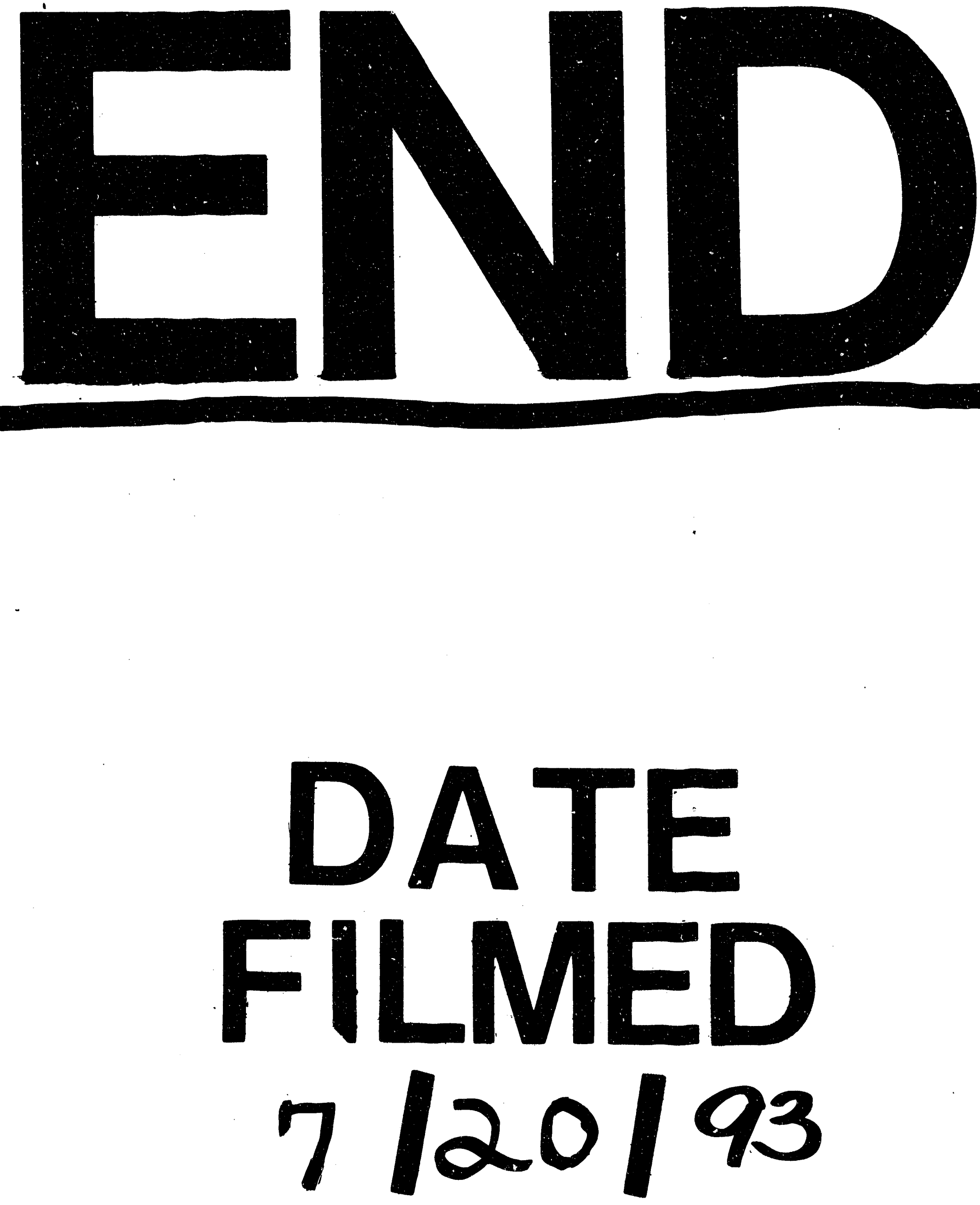
